\title{
Location Sharing Motivations of University Students
}

\section{Kemal Elciyar, Anadolu University, Turkey}

\begin{abstract}
Location sharing-check in- contains both geographical and semantic information about the visited venues, in the form tags. Many social networks like Facebook, Twitter, İnstagram, allow people to share location but main feature of Swarm and Foursquare is that.

There are many motivations have investigated for identifying location based services uses: inform each other, keep track of places, appear cool, get a reward etc. The aim of this study is examining user motivations of locations based services; Swarm and Foursquare. For this purpose, a survey has been applied to university students. Results suggest that location sharing applications supply user's need of location based socialization.
\end{abstract}




\section{Introduction}

Location sharing applications have not a long history in research. Location sharing-check incontains both geographical and semantic information about the visited venues, in the form tags. Foursquare, Swarm etc. are location-based social network (LBSN) and allow people to share their physical location with members of their social network. İn addition, Swarm and Foursquare also have gaming features and enables people to leave geotagged messages attached to locations. Many social networks like Facebook, Twitter, İnstagram, allow people to share location but main feature of Swarm and Foursquare is that. Foursquare and Swarm which has released by same corp. is a mobile social networking application with gaming elements that is built around people's check-ins. Unlike Foursquare Dodgeball used SMS to share location. Dodgeball has spawned other imitators and Foursquare was far away from the only LBSN when it released in 2009. Whrrl, Gowalla, Loopt, Brightkite are another application for this purpose. Foursquare's success is important because of facing with two Internet giants: Facebook-Facebook and Google- Latitude.

Foursquare and Swarm are not just a location based application, they are location-based social networking application, representing the merging of mobile communication and mobility with elements of online SNS. These applications enable new ways for people to connect with others, maintaining existing relationships, present themselves to others, and engage in participatory surveillance.

The check in was key for location based social networks. Swarm and Foursquare are a mobile social networking application with game elements that is built around user's check-ins. When using Foursquare and Swarm people go to locations and share those locations with their networks through check in process, like how people share contents and status updates with friends on social networking sites, Facebook etc. Users get points and badges for checking in and can become the mayor of a location if they checked in there the most times. In other words, users can both share their location in a social way and compete with themselves and others over who can earn the most points.

\section{Location Sharing and Applications}

From the traditional, automated approach of location-tracking, location sharing services now place the control with the user providing social media- oriented micro updates on their 
location. In addition, these services feature an interesting mix of public and private sharing, in which individual users may keep their location private or share with friends only, but in which all user generated venues are currently visible to all users (Cramer at al., 2011).

Location sharing services are defined as "a subset of web services meant to provide functions that are location-aware, where the use of such services is predicated on knowledge of where the services are engaged" (Wilson, 2012). Foursquare describes its services as a "mobile application that makes cities easier to use and more interesting to explore. İt is a friend-finder, a social city guide and a game that challenges users to experience new things, and rewards them for doing so. Foursquare let users 'check-in' to a place when they are there." (Lindqvist et al., 2011). Swarm and Foursquare are available for smartphones like IPhone, Android platform etc.

Foursquare-Swarm allow users to check-in at venues, write tips about there, upload contents like picture and video. Like other social networks Foursquare and Swarm users can easily make friend and monitor their friend locations and status. The role of geography and location in social networks has recently attracted increasing attention (Cheng et al., 2011).

Mobile check-ins are one of the most popular activities among young's and adults who go online with smart phones (Pew Internet Research, 2013). With the growth of mobile phone user number location sharing services has become a trend in social networks.

Game condition of foursquare presents virtual rewards for check-ins. Virtual rewards come in the forms of points, badges and mayorships visible in profile. People able to share their location with very large population bot through the location sharing services themselves and by moving check-in to their Twitter and Facebook pages. Location sharing applications allows people to know the location of others. Online social networks like Facebook, Instagram, Swarm allow users to share location information with others. Such a feature is quite popular, as \%30 of users attach locations to their posts (Bilogrevic et al., 2015).

There are many applications for location sharing. Connecto allowed users to tag locations and then automatically share it. Loccacino let for continuous updating of location based on specification of the people. Google Latitude mainly use GPS tracking model. Contrary to this 
many of the most popular location sharing services like Foursquare-Swarm, Facebook Place and Gowalla use one to many check-in model (Cramer at al., 2011).

When a user of Swarm or Foursquare want to share its location, it check in to a place using the mobile application or web site. Users of application can choose from a list of places nearby or search for a name or create a new place point. Users can also move their location sharing to their social media profiles. When a user checks in a place, a check in notification is by default pushed to their Foursquare and Swarm contacts. After check-in users see which other people checked in same place and total number of check-ins. Users can hide their checkins but all venues created by users are visible for all users. These applications provide gamification components like badged, points and mayorships to increase participation. Users have a publically accessible profile page, they can choose to use their real name or links of social pages. In Turkey young's use location sharing many times in the day actively due to many motivations. Following maps illustrates check-ins in Turkey and our case city Eskisehir:
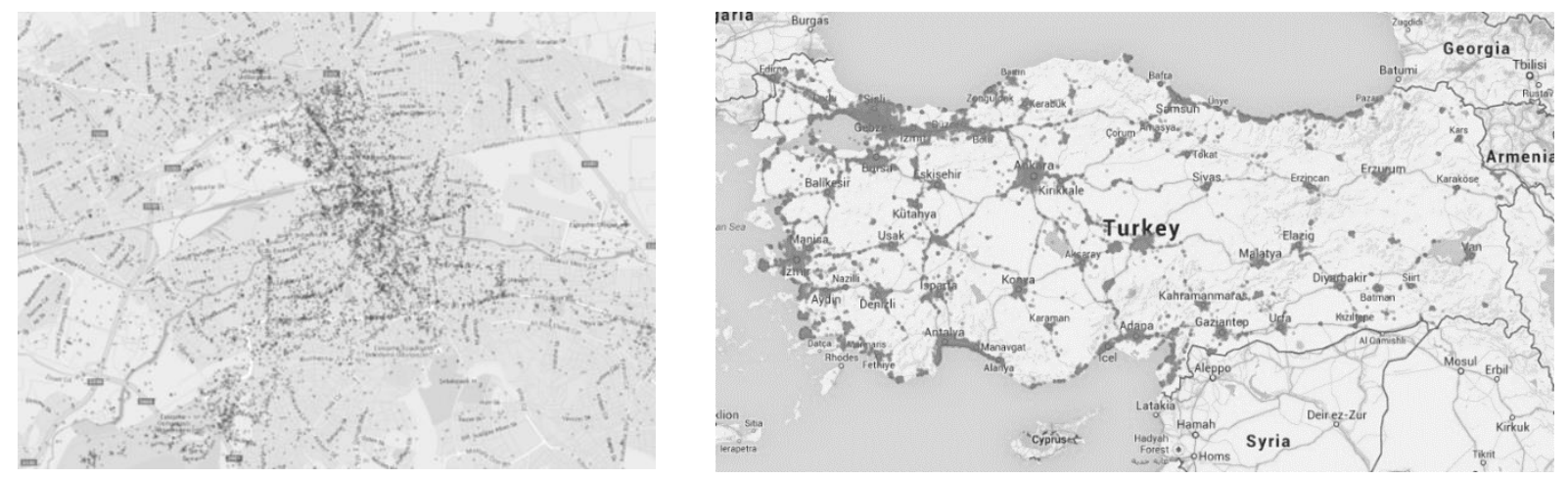

As it is seen in maps, Foursquare-Swarm users concentrates in large cities because they provide more opportunities and venues for young's.

\section{Motivations of Location Sharing}

Brown et al. (2007) argue that location sharing is not only practically sharing location or one's activity but also it is an emotional and moral affair. Location services can express whereabouts, furthermore portray users moods, lifestyle and events. By sharing location, users can exchange enjoyments and friendship and interact with social circle. Lindqvist et al. 
(2011) specified location sharing motivations including games and badges, social connection, place discovery and keep track for a venue. The motivation of location sharing include the wish to connect social circles and project an interesting image of themselves rather than geographical information. They found that people checked in at their location via Foursquare-Swarm in order to pursue continued connection with friends, enjoy the sharing of their locations, and finally to explore new places for their own experiences.

In the last period several studies has been applied to investigate users' motivations for share their locations in online social networks. Patil et al., (2012) applied two studies and results show that users' main motivations for sharing location included the desire to tell friends that they like a place to keep their social circle informed of where they are, to record their visits and appear cool and interesting rather than pointing to geographical location. In the same way results illustrated that social connections and impression management play an essential role in location sharing of Foursquare users.

Wang and Stefanone (2013) argued that presentation of place would facilitate and satisfy one's desire to be connected to other people online using an image of the person like offline social activities. Nov et al. (2009) described user motivations for Flickr in four dimensions: enjoyment, commitment to the community, self-development and reputation building. Some scholars use these dimensions for location sharing motivations.

\section{Methodology}

\section{Data Collection}

A secure online survey was created via Google Forms. One method used to collect participants was the use of social networking sites (SNSs), Facebook and Swarm. Study participants were offered information regarding the survey with a Google link they could follow to participate in the research effort. Participants were invited to share the link on their respective SNSs. Participants were able to complete the survey on any computer with Internet access, 24 h per day, Data collection was conducted from April 1 to April 20 of 2016. Before beginning the survey, participants read and indicated their consent to participate. The survey took an average of $3 \mathrm{~min}$ to complete. Data were pulled from the Google website and was downloaded on to a spreadsheet. Data were then examined for incomplete surveys. Once data were cleaned for analysis, a total of 105 participant responses were used in data analysis for 
the purpose of this research. The data were downloaded from the Google Forms website into a Microsoft excel file and imported into the statistical analysis program SPSS to test research hypotheses.

\section{Measures}

This study explored many variables probable in being significant determinants for motivations intention to use location sharing applications. An online survey to gain more insight into the motivations for 'checking in' has applied. A total of five motivation factors were tested, entailing the following: badges and fun, social connection, place discovery and keep tracking for place, gaming and motivator generating from others. Demographic factors of gender, age, mobile phone and check-ins were also tested. The researchers used the questionnaire which developed from Lindqvist (2011)'s study to illustrate the level of location sharing application use and motivations. Questionnaire has 20 items which includes likert scale of 1 to 5 and 6 demographic questions.

\section{Results}

\begin{tabular}{lrr}
\hline Sex & f & Percent \\
\hline Male & 46 & 43.8 \\
\hline Female & 59 & 56.2 \\
\hline Total & 105 & 100
\end{tabular}

Considering the range of participants by gender, women appear to be more. Women constitute $\% 56.2$ of the sample, men $\% 43.8$.

\begin{tabular}{lrr}
\hline $\begin{array}{l}\text { Operating } \\
\text { System }\end{array}$ & f & Percent \\
\hline IOS & 50 & 47.6 \\
\hline Android & 55 & 52.4 \\
\hline Total & 105 & 100 \\
\hline
\end{tabular}

When asked the operating system of the phone to the participants, $\% 47$ of participants answered IOS, \%52.4 Android. 


\begin{tabular}{|c|c|c|}
\hline \multicolumn{3}{|c|}{ Most Checked in Venues } \\
\hline & f & Percent \\
\hline Home & 2 & 1.9 \\
\hline School & 27 & 25.7 \\
\hline Cafe & 66 & 62.9 \\
\hline Night Clubs & 10 & 9.5 \\
\hline Total & 105 & 100 \\
\hline
\end{tabular}

Results illustrate that most people do not check-in when seeing a doctor. Note that for homes, the majority of people say that they never check-in at homes. Cafe is fairly popular places to check-in at. This finding is not too surprising, because the sample of the study consisted of students and due to the same reason many participants check-in when they are in school.

\begin{tabular}{|c|c|c|}
\hline $\begin{array}{l}\text { Means of Daily Check } \\
\text { İns }\end{array}$ & f & Percent \\
\hline 1 & 24 & 22,9 \\
\hline 2 & 29 & 27,6 \\
\hline 3 & 23 & 21,9 \\
\hline 4 & 12 & 11,4 \\
\hline 5 & 10 & 9,5 \\
\hline 6 & 4 & 3,8 \\
\hline 7 & 1 & 1,0 \\
\hline 10 & 1 & 1,0 \\
\hline 15 & 1 & 1,0 \\
\hline Total & 105 & 100,0 \\
\hline
\end{tabular}

Majority of participants check-in not too many in a day. Only $\% 7$ of participants check in more than 5 in a day period. 
Factor 1: Badges

\section{Survey Item}

I pay attention to the badges that I earn

I pay attention to the badges that others earn

I am happy for the badges I have earned

İ check in for getting badges
Item Mean Loading

$\begin{array}{ll}1.80 & .825\end{array}$

$1.34 \quad .731$

$1.81 \quad .872$

1.45

First factor of study is badges and scores about this factor are fairly low. According to results participants didn't deal with the badges and there is no correlation between their use of Swarm-Foursquare and badges. They don't feel happy because of badges and don't pay attention to them.

\section{Factor 2: Social connection}

\begin{tabular}{lrrr}
\hline Survey Item & Item Mean & Loading \\
\hline I use Swarm-Foursquare to let other people know that I am & 3.84 & .607 \\
available to hang out. & & \\
\hline Swarm-Foursquare helps me keep in touch with my friends. & 3.89 & .674 \\
\hline Swarm-Foursquare is fun because my friends are using it. & 3.80 & .752 \\
\hline I use Swarm-Foursquare to coordinate with my friends. & 3.67 & .607 \\
\hline I think Swarm-Foursquare is fun & 3.27 & .572 \\
\hline
\end{tabular}

If we consider location sharing applications as a social media, it is expected that high scores of social connection. Results confirm this hypothesis. The highest scores of study has seen in social connection factor. Young's use location sharing applications primarily for keep in touch with their friends and coordinate when they wish to meet. 


\section{Factor 3: Place discovery}

\section{Survey Item}

I have found a good tip about a place by using Swarm-

Foursquare.

Swarm-Foursquare has motivated me to go to new places.

I have discovered new places from my use of Swarm-

Foursquare.

I use Swarm-Foursquare to keep track of places I have visited.

İ look comments about a venue on Swarm-Foursquare
Item Mean Loading

$3.44 \quad .740$

$3.64 \quad .804$

$\begin{array}{ll}3.68 & .850\end{array}$ .831

$3.74 \quad .890$

Third factor of our study is place discovery and scores about this factor are fairly high. Results suggested that location sharing applications carry out their primarily objective of their nature. These applications mostly use for place discovering and tracking by participants. One visit a new venue wants to read comments of previous visitors and learn their experience in there. Participants also find out new places nearby and be motivated for discover them.

\section{Factor 4: Motivators generating from others}

\begin{tabular}{lrr}
\hline Survey Item & Item Mean & Loading \\
\hline I pay attention to other people's check-ins. & 2.44 & .762 \\
\hline I use foursquare because I can get discounts and special offers. & 1.82 & .625 \\
\hline I meet new people via Foursquare-Swarm & 2.65 & .722 \\
\hline I think my check ins show me more cool and fun. & 2.77 & .513
\end{tabular}

Factor 4 involves four items which can be define "motivators generating from others" like meeting new people or paying attention for others check-ins, but results suggested that participants' use of location sharing application don't correlate with motivators generating from others. In other words, participants use location sharing applications for meeting one's own needs. They don't care about discounts, new people or try to have a cool look. 
Factor 5: Game with yourself

\begin{tabular}{lrr}
\hline Survey Item & Item Mean & Loading \\
\hline I consider Swarm-Foursquare to be a game I play alone. & 2.06 & .800 \\
\hline $\begin{array}{l}\text { I use foursquare because it gives me something to do when I am } \\
\text { bored }\end{array}$ & 2.67 & .705 \\
\hline
\end{tabular}

Last factor of study is gaming and according to results participants don't think SwarmFoursquare as a game. They maybe sometimes use it when they get bored but gaming scores are not high for making deduction.

\section{Conclusion}

Study's findings support those of past studies of location sharing applications. Location sharing application use by participants as it's reason for being. Participants use location sharing application mostly tracking and discovering new venues. Furthermore they want to keep in touch with their friends via these apps. In sum, location sharing applications supply user's need of location based socialization. New studies may examine why people don't use these apps. By this way users and non-users can compare. 


\section{References}

Bilogrevic, I., Huguenin, K., Mihaila, S., Shokri, R., \& Hubaux, J. P. (2015). Predicting Users" Motivations behind Location Check-Ins and Utility Implications of Privacy Protection Mechanisms. In 22nd Network and Distributed System Security Symposium (NDSS" 15) (No. EPFL-CONF-202202).

Brown, B., Taylor, A. S., Izadi, S., Sellen, A., Jofish'Kaye, J., \& Eardley, R. (2007). Locating family values: A field trial of the Whereabouts Clock (pp. 354-371). Springer Berlin Heidelberg.

Cheng, Z., Caverlee, J., Lee, K., \& Sui, D. Z. (2011). Exploring Millions of Footprints in Location Sharing Services. ICWSM, 2011, 81-88.

Cramer, H., Rost, M., \& Holmquist, L. E. (2011, August). Performing a check-in: emerging practices, norms and'conflicts' in location-sharing using foursquare. In Proceedings of the 13th International Conference on Human Computer Interaction with Mobile Devices and Services (pp. 57-66). ACM.

Lindqvist, J., Cranshaw, J., Wiese, J., Hong, J., \& Zimmerman, J. (2011, May). I'm the mayor of my house: examining why people use foursquare-a social-driven location sharing application. In Proceedings of the SIGCHI conference on human factors in computing systems (pp. 2409-2418). ACM.

Nov, O., Naaman, M., \& Ye, C. (2010). Analysis of participation in an online photo-sharing community: A multidimensional perspective. Journal of the American Society for Information Science and Technology, 61(3), 555-566.

Patil, S., \& Lai, J. (2005, April). Who gets to know what when: configuring privacy permissions in an awareness application. In Proceedings of the SIGCHI conference on Human factors in computing systems (pp. 101-110). ACM.

Wang, S. S., \& Stefanone, M. A. (2013). Showing off? Human mobility and the interplay of traits, self-disclosure, and Facebook check-ins. Social Science Computer Review, 0894439313481424.

Wilson, M. (2012). Location-based services, conspicuous mobility, and the locationaware future. Geoforum, 43, 1266e1275. 